Return to the Manage Active Submissions page at http://spie.org/submissions/tasks.aspx and approve or disapprove this submission. Your manuscript will not be published without this approval. Please contact author_help@spie.org with any questions or concerns.

\title{
THz carrier dynamics and magnetotransport study of topological surface states in thin film Bi2Se3
}

\author{
Varun S. Kamboj ${ }^{* a}$, Angadjit Singh ${ }^{a}$, Thierry Ferrus ${ }^{b}$, Harvey E. Beere ${ }^{a}$, Liam B. Duffy ${ }^{c}$, Thorsten \\ Hesjedal ${ }^{c}$, Crispin H.W. Barnes ${ }^{a}$ and David A. Ritchie ${ }^{a}$
}

${ }^{\mathrm{a} C a v e n d i s h ~ L a b o r a t o r y, ~ U n i v e r s i t y ~ o f ~ C a m b r i d g e, ~ J . ~ J . ~ T h o m s o n ~ A v e n u e, ~ C a m b r i d g e ~ C B 3 ~ 0 H E, ~}$ United Kingdom;

${ }^{\text {b} H i t a c h i ~ C a m b r i d g e ~ L a b o r a t o r y, ~ J . ~ J . ~ T h o m s o n ~ A v e n u e, ~ C a m b r i d g e ~ C B 3 ~ O H E, ~ U n i t e d ~ K i n g d o m ; ~}$ ${ }^{c}$ Department of Physics, Clarendon Laboratory, University of Oxford, Oxford OX1 3PU, United Kingdom;

\begin{abstract}
The surface of a topological insulator harbors exotic topological states, protected against backscattering from disorder by time reversal symmetry. The study of these exotic quantum states not only provides an opportunity to explore fundamental phenomena in condensed matter physics, such as the spin Hall effect, but also lays the foundation for applications from quantum computing to spintronics. Conventional electrical measurements suffer from substantial bulk interference, making it difficult to clearly distinguish topological surface states from bulk states. Employing terahertz time-domain spectroscopy, we study the temperature-dependent optical behavior of a 23-quintuple-thick film of bismuth selenide $\left(\mathrm{Bi}_{2} \mathrm{Se}_{3}\right)$ allowing for the deconvolution of the surface state response from the bulk. Our measurement of carrier dynamics give an optical mobility exceeding $2100 \mathrm{~cm}^{2} / \mathrm{V} \cdot \mathrm{s}$ at $4 \mathrm{~K}$, indicative of a surface-dominated response, and a scattering lifetime of $\sim 0.18 \mathrm{ps}$ and a carrier density of $6 \times 10^{12} \mathrm{~cm}^{-2}$ at $4 \mathrm{~K}$ for the $\mathrm{Bi}_{2} \mathrm{Se}_{3}$ film. The sample was further processed into a Hall bar device using wet chemical etching and compared with $\mathrm{Ar}^{+}$ion milled film, which resulting in a reduced Hall mobility from milling. The magneto-conductance transport reveals weak antilocalization behavior in our $\mathrm{Bi}_{2} \mathrm{Se}_{3} \mathrm{sample}$ consistent with the presence of a single topological surface state mode.

Keywords: topological insulators, bismuth selenide, terahertz spectroscopy, optical mobility, weak antilocalization.
\end{abstract}

\section{INTRODUCTION}

Topological insulators (TIs) are a new class of quantum materials that have an insulating bulk but possess a gapless conducting surface state arising from the non-trivial topology of the band structure ${ }^{1}$. The topological surface state (TSS) is 'robust' from protection against backscattering by spin-momentum locking and time reversal symmetry ${ }^{1-4}$. The surface state of a three-dimensional TI involves an odd number of Dirac cones in the surface Brillouin zone, guaranteed by the $\mathrm{Z}_{2}$ topological invariant of the bulk ${ }^{4,5}$. The surface state of $\mathrm{Bi}_{2} \mathrm{Se}_{3}$ presents a particularly special case with a single Dirac cone on the surface ${ }^{6-9}$, which can be described by strong spin orbit coupling ${ }^{5,10}$ that exhibits helical behavior ${ }^{11}$, i.e., spin and translation momentum vectors are at fixed angles $\left(90^{\circ}\right)$ to each other. The surface state, characterized by their topological order, makes the electron motion resistant against scattering by non-magnetic impurities. Such a dissipationless character of carriers is suggested to provide novel applications in technology, for instance in spintronics ${ }^{1,7,12}$ or quantum computing ${ }^{13}$. In an ideal TI, the bulk does not contribute to charge transport and the conductivity is solely governed by surface carriers. However, the present generation of TIs, in particular the chalcogenides of the $(\mathrm{Bi}, \mathrm{Sb})_{2}(\mathrm{Se}, \mathrm{Te})_{3}$ family, exhibit significant contributions from the bulk carriers to the net conductivity. The primary reason for this behavior is the doping due to Se or Te vacancies and anti-site defects ${ }^{14}$, which shifts the Fermi level close to the bulk conduction band, in the case of $\mathrm{Bi}_{2} \mathrm{Se}_{3}$. The discrepancy between the bulk and surface carrier density can cause band bending (with an accumulation layer) resulting in the formation of a two-dimensional electron gas (2DEG), if the Fermi level crosses the bulk band near the surface. Such a 2DEG is limited to within $\sim 20 \mathrm{~nm}$ of the surface ${ }^{15,16}$ and appears alongside the TSS. The usability of TIs as a viable material for novel technological applications requires unambiguous separation of the surface state response

*E-mail: vk302@cam.ac.uk, Phone: +44 1223768142

10531 - 18 V. 5 (p.1 of 10) / Color: No / Format: A4 / Date: 1/21/2018 3:39:24 PM

SPIE USE: DB Check, Prod Check, Notes: 
Return to the Manage Active Submissions page at http://spie.org/submissions/tasks.aspx and approve or disapprove this submission. Your manuscript will not be published without this approval. Please contact author_help@spie.org with any questions or concerns.

from the interfering bulk states. The TSS differs fundamentally from the bulk states in their topological origin, and hence exhibit much higher scattering lifetime and mobilities owing to their topological protection ${ }^{3}$. However, conventional electrical transport measurements face the difficultly of probing the TSS exclusively, and distinguishing it from the bulk carrier contribution. While angle-resolved photoemission spectroscopy (ARPES) can probe the TSS in a TI directly, it lacks the energy resolution to resolve energy states near the Fermi level, due to x-ray photoexcitation of the carries. ${ }^{17}$ Recently, using terahertz time-domain spectroscopy (THz-TDS) on $\mathrm{Bi}_{1.5} \mathrm{Sb}_{0.5} \mathrm{Te}_{1.8} \mathrm{Se}_{1.2}$ (BSTS), ${ }^{18}$ Tang et al. reported a combination of surface and bulk response due to issues associated with defects and surface deterioration. In contrast, $\mathrm{Bi}_{2} \mathrm{Se}_{3}$ has a relatively stable surface with negligible reactivity to oxygen ${ }^{19}$. It would be highly desirable to have a technique which can detect and isolate the TSS response from the bulk unambiguously, close to the Fermi level, with the capability to selectively address the TSS. Terahertz (THz) spectroscopy is a contact-free optical technique, used to probe the low-energy excitations in strongly correlated electron gases and low dimensional systems. In the THz (sub-millimeter) region, the frequency of the electromagnetic radiation is comparable to the carrier damping rate $\left(10^{-13} \mathrm{~s}^{-1}\right)$, rendering this technique particularly sensitive to study the TSS in TIs.

In this work, we describe the use of a broadband THz-TDS to unambiguously identify the TSS contribution, and to distinguish it from the bulk contributions, by measuring the $\mathrm{THz}$ transmittance of $\mathrm{Bi}_{2} \mathrm{Se}_{3}$ as a function of temperature. The carrier scattering lifetime is extracted entirely from the phase of the $\mathrm{THz}$ signal at different temperatures, which further obtains the mobilities and carrier densities by purely optical means, allowing a complete characterization of the TSS response. It is worth stating that our technique does not require an optical pump arrangement ${ }^{17,20}$ conventionally used to obtain parameters such as scattering lifetime. Instead, it allows for a more straightforward measurement of the equilibrium (non-photoexcited) material properties, especially relevant for TI-based electronic applications. The sample was further processed into a Hall bar using wet chemical etching, allowing for a comparison between the optical and electrical measurements. Furthermore, the magnetotransport measurements reveal the value of a prefactor, $\alpha$, corresponding to the number surface states as 0.5 , indicating that the observed response could originate from top TSS or a coupled top and bottom TSS. The work provides fundamental insights into the carrier dynamics of the TSS and bulk carriers using a noninvasive method, enabling a technique to characterize future TI material system for novel future applications.

\section{TERAHERTZ RESPONSE OF TI-FILM}

The complex optical transmission, $\tilde{T}(\omega)=T_{1}+i T_{2}$, of a thin film allows for the determination of the mobilities and carrier densities, directly comparable to values obtained from electrical transport, where $T_{1}$ and $T_{2}$ are the real and imaginary components, respectively. The imaginary and real components of the transmission are independent of each other, albeit
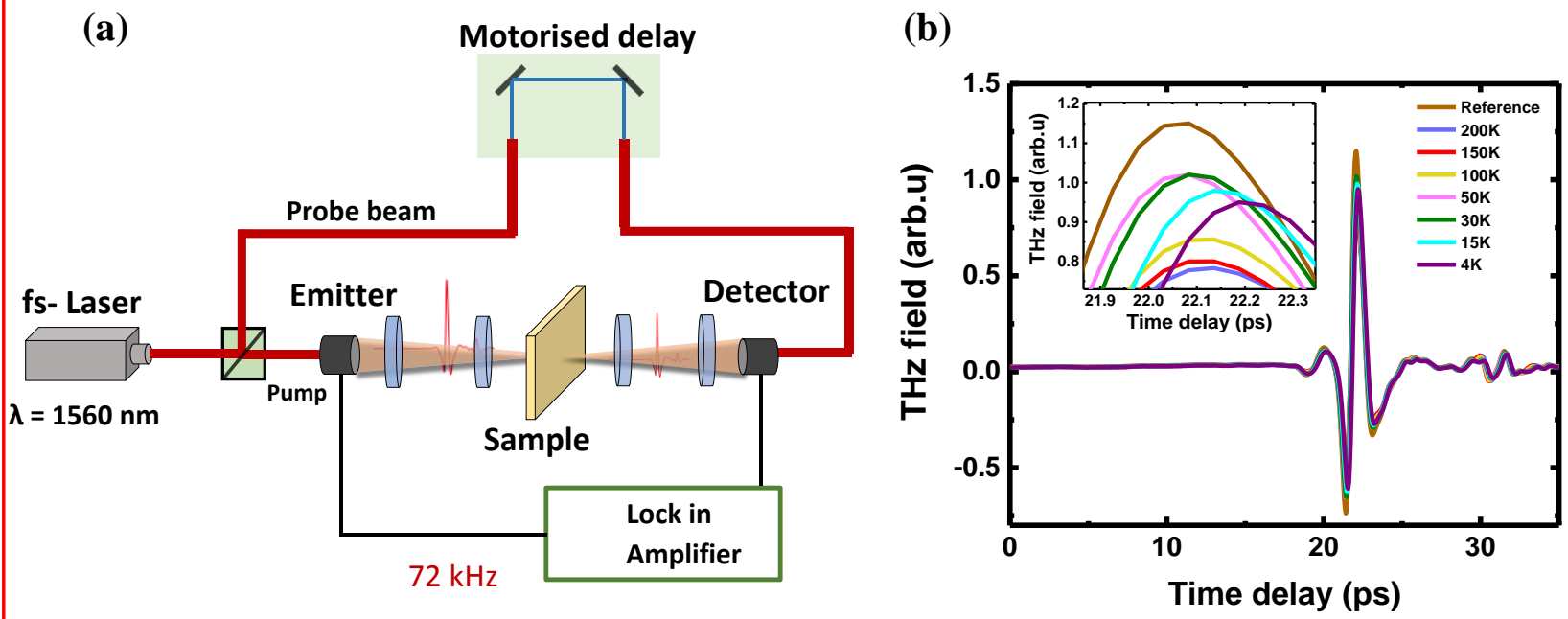

Figure 1. (a) Schematic of a THz-TDS optical setup. A 60-mW pump laser output of 90 fs duration, centered at $1560 \mathrm{~nm}$, was split into two paths for $\mathrm{THz}$ generation (pump) and detection (probe). (b) Time domain picosecond (ps) pulse response transmitted through $\mathrm{Bi}_{2} \mathrm{Se}_{3}$ film of $23 \mathrm{QL}$ (quintuple layer) thickness at different temperatures. The inset shows a magnified pulse response transmitted through the $\mathrm{Bi}_{2} \mathrm{Se}_{3}$ film. The reference is the transmission through a blank (0001)-oriented sapphire substrate, obtained at $200 \mathrm{~K}$. All the temperature measurements were performed in a cryostat with optical access with a base temperature of $4 \mathrm{~K}$. 
Return to the Manage Active Submissions page at http://spie.org/submissions/tasks.aspx and approve or disapprove this submission. Your manuscript will not be published without this approval. Please contact author_help@spie.org with any questions or concerns.

being measured simultaneously in a THz-TDS measurement. The imaginary part of the transmission is associated with the carrier scattering which phase shifts the THz response with respect to a reference substrate, and hence can be used to obtain the momentum relaxation time and mobility of carriers in the TI film. This makes THz-TDS a powerful tool to measure key material parameters related to TI thin films. A schematic of the optical arrangement is illustrated in Figure 1a. Pulses from a $60-\mathrm{mW}$ pump laser of $90 \mathrm{fs}$ duration at $1560 \mathrm{~nm}$ (repetition rate of $100 \mathrm{MHz}$ ) are split into two paths: the first pump component focused down to a $40 \mu \mathrm{m}$ spot onto the THz emitter, resulting in a broadband THz emission with a spot size of $\sim 1 \mathrm{~mm}$; the second probe component is passed through the delay stage ultimately leading to the gated $\mathrm{THz}$ detection. The sample was mounted in a Janis continuous flow cryostat with optical access with a base temperature of $4 \mathrm{~K}$, to obtain a temperature-dependent $\mathrm{THz}$ response.

The time-resolved $\mathrm{THz}$ transmission through the $\mathrm{Bi}_{2} \mathrm{Se}_{3}$ thin film was measured at various temperatures as shown in Figure $1 \mathrm{~b}$, together with a magnified pulse response shown in the inset. We observe that the transmitted $\mathrm{THz}$ field intensity initially increases with reducing temperature down to $30 \mathrm{~K}$ (see inset in Figure 1b), due to a reduced free carrier absorption, implying the freezing out of bulk charge carries in $\mathrm{Bi}_{2} \mathrm{Se}_{3}$. However, below $30 \mathrm{~K}$ (and down to $4 \mathrm{~K}$ ), the transmitted intensity reduces with decreasing temperature, indicating that the carriers have nature distinctly different from the $\mathrm{Bi}_{2} \mathrm{Se}_{3}$, bulk carriers, with different properties and features. Such behavior is expected, since reducing the temperature (from 200 K) should lead to a reduced contribution from the bulk states, thus leaving the TSS (consisting mainly of the top and bottom contributions in an extended thin film system) as the main contributor to the conductance. Therefore, a drop in the $\mathrm{THz}$ transmission is indicative of a transition to TSS-dominated transport below $30 \mathrm{~K}$ in our film. ${ }^{46}$

To establish the distinct signature of the TSS at low temperatures we can obtain the scattering lifetime and mobility of $\mathrm{Bi}_{2} \mathrm{Se}_{3}$. Using Tinkham's equation for thin films ${ }^{21}$ and substituting for the complex Drude conductivity as $\tilde{\sigma}(\omega)=\sigma_{0} /(1-$ $i \omega \tau)$, we obtain the following relationship between the imaginary and the real transmission: $\operatorname{Im} .\{\widetilde{T}(\omega)\} / \operatorname{Re} .\{\tilde{T}(\omega)\}=$ $-\omega \tau=\tan \phi$. Here, $\phi$ is the phase angle between the sample and substrate waveform, $\omega$ is the angular frequency and $\tau$ is the scattering lifetime for the carriers. Thus, the scattering lifetime can be directly obtained from the imaginary and the real part of the transmission, without the need of any further parameters.


Figure 2. (a) Plot of $\tan \phi$ vs. frequency. The solid lines represent the linear fit to each data set. (b) Scattering lifetime $\tau$ deduced from the THz-TDS. The solid line represents the power law dependence of $\tau \sim T^{-1.4}$, which suggests a dominance of the phonon scattering mechanism at temperatures above $50 \mathrm{~K}$

Figure 2a shows a plot of $\tan \phi$ as a function of frequency, with a liner regression, with its slope directly yielding the scattering lifetime $(\tau)$ and the mobility $(\mu)$ as a function of temperature, where $\mu=\tau e / \mathrm{m}^{*}$. As a first-order approximation, the electron effective mass $m^{*}$ in $\mathrm{Bi}_{2} \mathrm{Se}_{3}$ is $0.15 m_{e}$, where $m_{e}$ is the bare electron mass. ${ }^{22-24}$ The scattering lifetimes of carriers exhibits a power law dependence of $\tau \sim T^{-1.4}$ at temperatures above $50 \mathrm{~K}$, consistent with the phonon scattering mechanism expected at higher lattice temperatures ${ }^{25}$ (Figure 2b). We obtained a scattering lifetime of $\tau=0.18$ ps at $4 \mathrm{~K}$, 
Return to the Manage Active Submissions page at http://spie.org/submissions/tasks.aspx and approve or disapprove this submission. Your manuscript will not be published without this approval. Please contact author_help@spie.org with any questions or concerns.

and thus the mean-free path of surface electrons as $l=\tau \cdot v_{F} \approx 119 \mathrm{~nm}$, where $v_{F}\left(\approx 6.6 \pm 3 \times 10^{5} \mathrm{~m} / \mathrm{s}\right)$ is the Fermi velocity of $\mathrm{Bi}_{2} \mathrm{Se}_{3}{ }^{7,26,27}$. An increase in the scattering lifetime (and mean-free path) with decreasing temperature strongly suggests a TSS associated response from the THz measurement. In the next section, we discuss the electrical transport measurements on the $\mathrm{Bi}_{2} \mathrm{Se}_{3}$ thin film, before comparing them with the $\mathrm{THz}$ response.

\section{MAGNETOTRANSPORT}

Magneto-transport measurements were carried out on the 23-QL-thick $\mathrm{Bi}_{2} \mathrm{Se}_{3}$ sample, which was processed in the form of Hall bars devices (of dimensions $1400 \mu \mathrm{m} \times 80 \mu \mathrm{m}$ ), to characterize the material properties such as mobility and carrier density. The Hall bar devices were fabricated using photolithography and wet chemical etching, to define a mesa with a length-to-width $(L / W)$ ratio of 5.25 . We started the process by cleaving a sample measuring $5 \mathrm{~mm} \times 5 \mathrm{~mm}$ from the sapphire substrate. Shipley 1813 photoresist was spun on the sample for $45 \mathrm{~s}$ at $5500 \mathrm{rpm}$. This is followed by baking the sample at $110^{\circ} \mathrm{C}$ for 2 minutes. The sample was then exposed to UV light for $6.5 \mathrm{~s}$ through a mask aligner and developed using MF319 developer for $30 \mathrm{~s}$ followed by a rinse in the DI water and dry $\mathrm{N}_{2}$. Then by using a wet chemical etching solution containing $\mathrm{HCl}, \mathrm{H}_{2} \mathrm{O}_{2}$, acetic acid and distilled water, in the ratio 5:4:40:80, the pattern was transferred into the $\mathrm{Bi}_{2} \mathrm{Se}_{3}$ film. The etch rate was $\sim 10 \mathrm{~nm} / \mathrm{min}$ confirmed using a Dektak profiler. Another sample was processed into Hall bar device using the $\mathrm{Ar}^{+}$ion milling. A successive photolithography step was used to define the source, drain and Hall contacts, following a similar process flow. A chlorobenzene dip for 2 minutes is used to harden the resist to give a good overhang during the evaporation and lift-off process. Finally, thermal evaporation was used to deposit titanium/gold (Ti/ $\mathrm{Au}-20 / 80$ $\mathrm{nm})$ to obtain the ohmic contacts which were then wire bonded to a leadless chip carrier. The resultant device is shown in the inset of Figure 3(a) was used to carry the Hall measurements.

Figure 3(a) shows the temperature-dependent longitudinal sheet resistance $\left(R_{\text {sheet }}\right)$. The sheet resistance shows a monotonic decrease, as the temperature is reduced from room temperature down to $50 \mathrm{~K}$, attributed to reduced electron-phonon scattering. ${ }^{28}$ However, close to $30 \mathrm{~K}$, the resistance flattens out indicating the freezing out of the bulk carriers. Below 30 $\mathrm{K}$, the transport is likely to be dominated by surface conduction and impurity states, in good agreement with previously reported results for a similar thickness of $\mathrm{Bi}_{2} \mathrm{Se}_{3}$ film. ${ }^{29}$ The field-dependent Hall resistance $\left(R_{x y}\right)$ at different temperatures was measured in an Oxford Instrument He-4 cryostat, as shown in Figure 2b. The cryostat is capable of reaching a base temperature of $1.5 \mathrm{~K}$ and a maximum magnetic field up to $9 \mathrm{~T}$. The Hall slopes can be used to deduce the carrier density over the temperature range of the measurement. The measurements were obtained using an a.c. lock-in four-terminal setup with an input current $\left(I_{\mathrm{SD}}\right)$ of $1 \mu \mathrm{A}$ at a frequency of $77 \mathrm{~Hz}$. The $R_{\text {sheet }}$ of the channel was $\sim 1.0 \mathrm{k} \Omega$ at room temperature. The Hall measurements ( $R_{\mathrm{xy}}$ vs $B$ ) show a certain degree of non-linearity (Figure $\left.3 \mathrm{~b}\right)$ at low temperatures $(<30 \mathrm{~K})$.

(a)

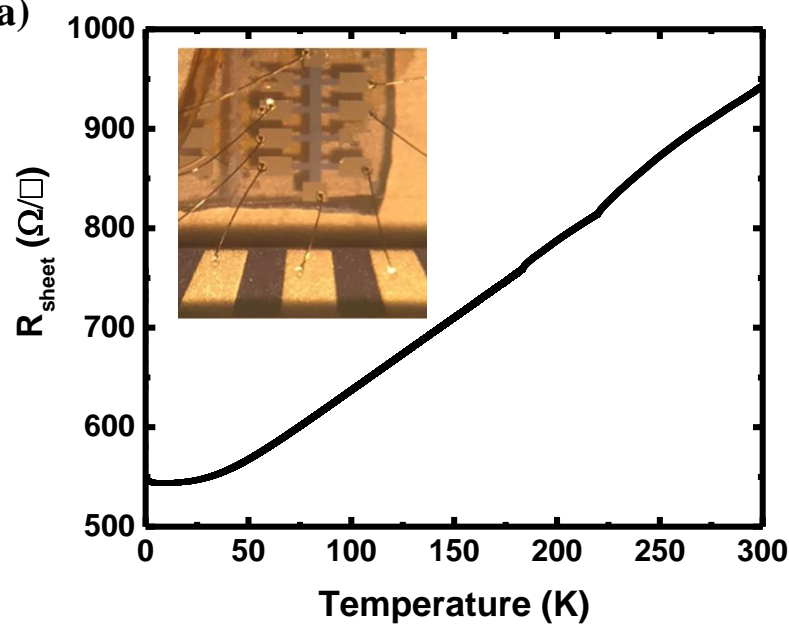

(b)

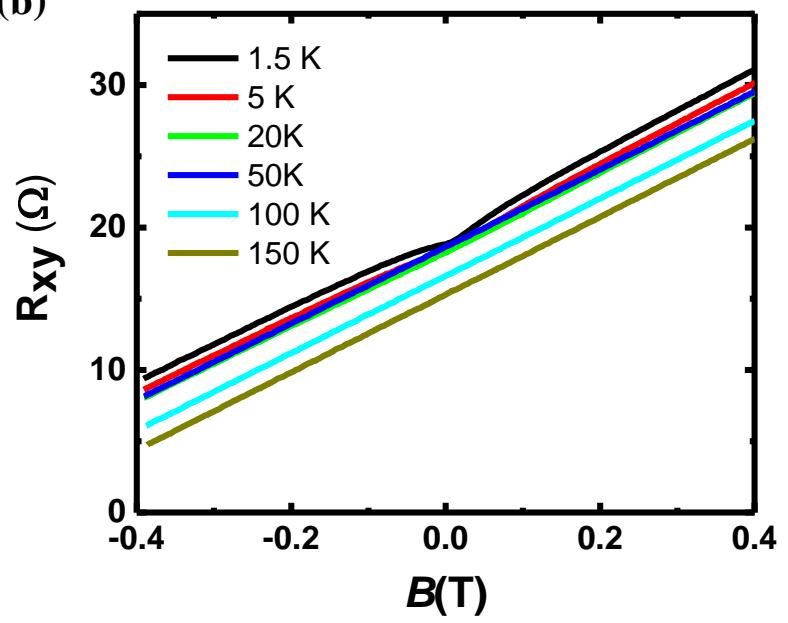

Figure 3. (a) The longitudinal (sheet) resistance obtained from the transport measurements. The inset shows an optical image of the Hall bar device. (b) Typical field dependence of Hall resistance $R_{\mathrm{xy}}$ for a range of temperatures for the 23-QL-thick Bi2 $2 \mathrm{Se}_{3}$ sample. 
Return to the Manage Active Submissions page at http://spie.org/submissions/tasks.aspx and approve or disapprove this submission. Your manuscript will not be published without this approval. Please contact author_help@spie.org with any questions or concerns.

Such non-linearity in $R_{\mathrm{xy}}$ is associated with the multiple contributions from the TSS and other non-topological bulk channels, in the transport measurements of $\mathrm{Bi}_{2} \mathrm{Se}_{3} .{ }^{29} \mathrm{We}$ note that the Hall resistance does not reduce to zero in zero field. This might be due to a finite value of the longitudinal contribution to the Hall measurement. We further obtained longitudinal sheet conductance ${ }^{2 \mathrm{D}} \sigma_{\mathrm{xx}}$ (or Sheet $\rho_{\mathrm{xx}}$ ) from transport measurements normalized to ${ }^{2 \mathrm{D}} \sigma_{\mathrm{xx}}$ at zero field. The results were plotted and fit to the Hikami-Larkin-Nagaoka (HLN) equation, resulting in parameters such as the number of surface conducting channels, $\alpha$, as discussed later. As the field was swept from $-0.4 \mathrm{~T}$ to $+0.4 \mathrm{~T}$, we observed a sharp increase in the conductivity at low magnetic field $B$, when increasing the temperature from $1.5 \mathrm{~K}$ to $50 \mathrm{~K}$ demonstrating weak antilocalization (WAL) behavior (Figure 4a). As in any quantum transport interference system, a phase coherence length $\left(L_{\varphi}\right)$, or phase relaxation time $\left(t_{\varphi}\right)$, between the incident and backscattered electron are defined as the length (or time) over which the electron wavefunction retains its phase and can interfere. An external magnetic field or magnetic impurities can destroy TRS and reduce the phase coherence length. Therefore, WAL effects are usually observed as a sharp peak in the conductance (dip in the resistance) with the application of a small magnetic field. More specifically, in the presence of an external perpendicular magnetic field, the time-reversed channels undergo destructive quantum interference resulting in a peak in magnetoconductance. Hence, such a phenomenon reflects both the Dirac nature of the surface states as well as the strong spin-orbit interaction characteristic of the TI material..$^{30}$

(a)

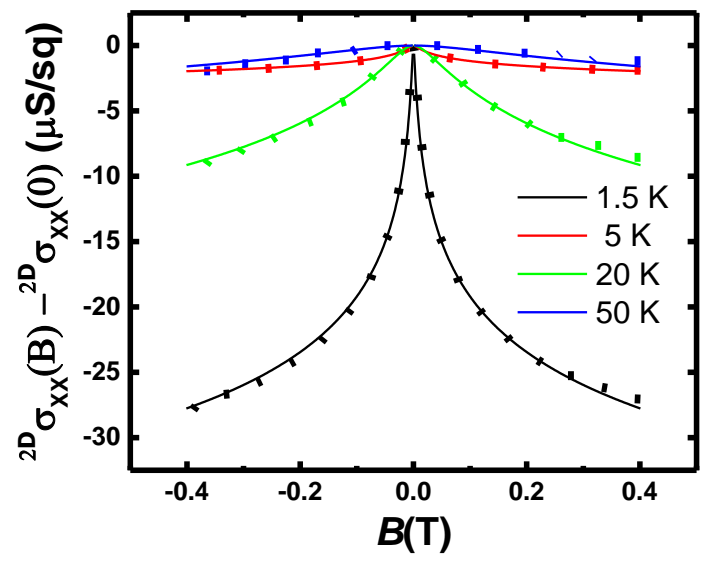

(b)

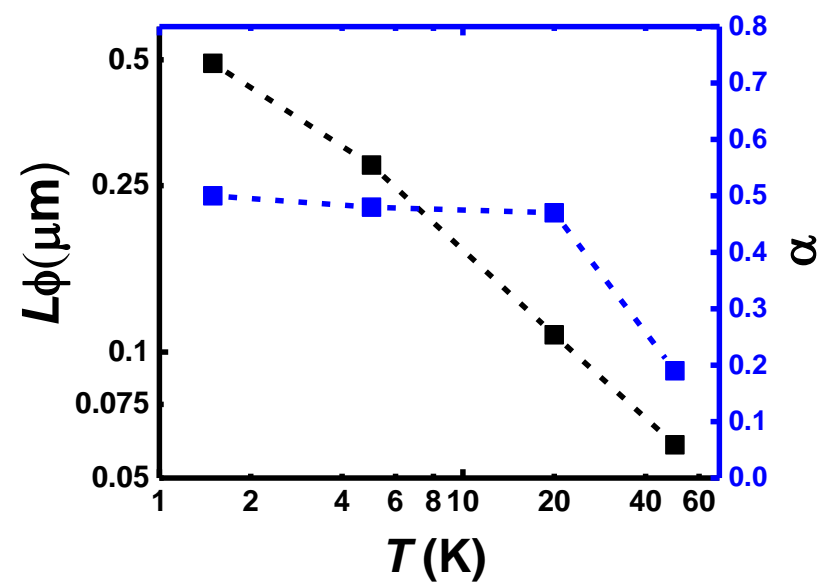

Figure 4. Weak antilocalization effect in a 23-QL-thick $\mathrm{Bi}_{2} \mathrm{Se}_{3}$ film. (a) Normalized conductivity changes as a function of magnetic field measured at different temperatures. Each data set is fitted (solid line) to the HLN formula described in the text. The cusp in conductance is a characteristic feature of the WAL effect. (b) Plot of the fitted parameters $L_{\varphi}$ and $\alpha$ as a function of temperature obtained from the WAL fit.

This behavior is described well by the Hikami-Larkin-Nagaoka (HLN) equation ${ }^{31}$ given as:

$$
\Delta \sigma_{x x}^{2 D} \equiv \sigma_{x x}^{2 D}(B)-\sigma_{x x}^{2 D}(0)=\alpha \frac{e^{2}}{2 \pi^{2} \hbar}\left[\ln \left(\frac{\hbar}{4 e B L_{\varphi}^{2}}\right)-\Psi\left(\frac{1}{2}+\frac{\hbar}{4 e B L_{\varphi}^{2}}\right)\right]
$$

where $\Delta \sigma_{x x}^{2 D}$ indicates the normalized 2D sheet conductivity, $B$ is the magnetic field perpendicular to the plane of the film, $e$ is the electronic charge, $\hbar$ is the reduced Planck constant, $\Psi$ is the digamma function, $\alpha$ is the number of topological surface modes (TSM), and $L_{\varphi}$ is the phase coherence length.

Figure $4 \mathrm{~b}$ shows the variation of $\alpha$ and $L_{\varphi}$ as a function of temperature, extracted from the fit of the HLN equation to the WAL signal. We obtain a value for the prefactor $\alpha \approx 0.5$, which remains largely unchanged from $1.5 \mathrm{~K}$ to $20 \mathrm{~K}$. Steinberg et al. ${ }^{32}$ demonstrated that application of a gate voltage can vary $\alpha$ between 0.5 to 1 and hence it can be used phenomenologically as a measure of the top and bottom channel separation (with $\alpha \approx 1$ indicating the decoupling of the 
Return to the Manage Active Submissions page at http://spie.org/submissions/tasks.aspx and approve or disapprove this submission. Your manuscript will not be published without this approval. Please contact author_help@spie.org with any questions or concerns.

top surface from the rest of the system). In this context, $\alpha \approx 0.5$ suggests that the top and the bottom TSS could be coupled in the $\mathrm{Bi}_{2} \mathrm{Se}_{3}$ film ${ }^{32}$. Another possible scenario is that the top surface is contributing significantly more to the WAL than the bottom surface, hence resulting in $\alpha \approx 0.5$. This might be due to a somewhat reduced phase coherence length, $L_{\varphi}$ at the bottom interface, owing to the defects present at the TI-substrate interface ${ }^{33,34}$, possibly also affected by the lattice mismatch between the $\mathrm{Bi}_{2} \mathrm{Se}_{3}$ and the substrate. ${ }^{44}$ Beyond $20 \mathrm{~K}, \alpha$ starts to drop with temperature indicating increasing bulk interference ${ }^{35}$. The sharpness of the WAL cusp determines the phase coherence length, $L_{\varphi}$. This length characterizes phase coherent behavior of carriers before an inelastic scattering event, indicating the quality of the film. The obtained $L_{\varphi}$ shows a strong temperature dependence as shown in Figure $4 \mathrm{~b}$. The coherence length increases with decreasing temperature, with a maximum value of $\sim 500 \mathrm{~nm}$ at $1.5 \mathrm{~K}$.

\section{MOBILITY AND CARRIER DENSITY: OPTICAL VS ELECTRICAL}

The scattering lifetime $(\tau)$ deduced in Section 2 can be used to further obtain the mobility $(\mu)$ as a function of temperature, where $\mu=\tau e / m^{*}$. Figure 5a shows the temperature-dependence of THz and Hall mobility, $\mu$ from $200 \mathrm{~K}$ to $4 \mathrm{~K}$. The plot of $\mathrm{THz} \mu$ vs. T, highlights two different regimes: above $50 \mathrm{~K}$, mobility drops gradually with increasing temperature, indicating that phonon scattering dominates any rise in the conduction due to increasing carrier density $(n)$, with the resulting conduction being primarily bulk-dominated. However, below $50 \mathrm{~K}$, the mobility rises more rapidly demonstrating a metallic surface state response. At low temperatures below $50 \mathrm{~K}$, the phonon scattering is alleviated with the freezing of bulk carriers, leaving the TSS response as the dominant contribution to the measured conduction, thus explaining the sharp rise in mobility (and the scattering lifetime, Figure $2 b$ ).

It is interesting to note that the mobility shows a gradual reduction above $50 \mathrm{~K}$, in contrast to its sharp rise below $50 \mathrm{~K}$. This arises from a unique non-trivial property of TIs where a Dirac-like surface state co-exists with the bulk bands ${ }^{3}$. Although, increasing the temperature increases the surface scattering ( $\tau$ decreases), the electronic relaxation from bulk to the TSS ${ }^{36,37}$ states suppresses this increase of the surface scattering rate, hence resulting in a smaller gradient in mobility at high temperatures $(>50 \mathrm{~K})$. However, at low temperatures $(<50 \mathrm{~K})$ the electronic relaxation from the bulk to the TSS is quenched $^{38}$, allowing a rapid change in the optical mobility. Such non-trivial behavior has been reported before in TIs, where electron relaxation from bulk to the surface suppresses the photo-induced surface scattering at high temperature ${ }^{39}$. The measured value of the surface state mobility at $4 \mathrm{~K}$ was $2124 \mathrm{~cm}^{2} / \mathrm{V} \cdot \mathrm{s}$ (with an effective mass of $\left.0.15 \mathrm{~m}_{e}\right)^{22}$ and $\sim 220$ $\mathrm{cm}^{2} / \mathrm{V} \cdot \mathrm{s}$ at $100 \mathrm{~K}$, consistent with the literature values for TSS and bulk mobilities ${ }^{29}$. The mobilities obtained from the Hall measurements are plotted alongside the THz optical mobilities (Figure 5a). The THz and Hall mobilities are relatively consistent at $50 \mathrm{~K}$ and above.

(a)

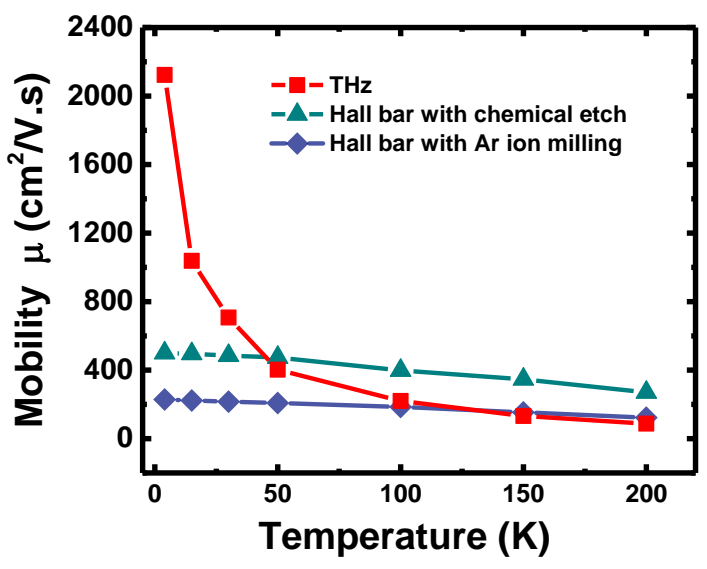

(b)

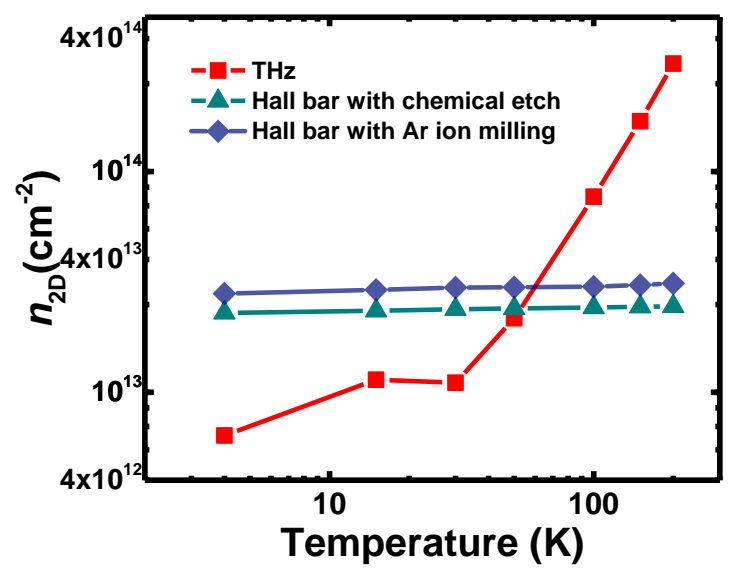

Figure 5. (a) The plot of mobility $\mu$ vs. temperature obtained from the THz measurements (red curve) demonstrates a metallic surface state response below $50 \mathrm{~K}$, and the Hall measurements (green curve) a predominantly bulk type response. (b) Sheet carrier density as a function of temperature deduced from the THz measurements (red curve), Hall measurements with wet chemical etch (green curve), Hall measurements with $\mathrm{Ar}^{+}$ion milling (purple curve). 
Return to the Manage Active Submissions page at http://spie.org/submissions/tasks.aspx and approve or disapprove this submission. Your manuscript will not be published without this approval. Please contact author_help@spie.org with any questions or concerns.

The discrepancy below $100 \mathrm{~K}$ highlight the difficulty in identifying the TSS with respect to the bulk contribution, as the Hall measurements primarily show a bulk response, which is attributed to substantially high surface scattering even at low temperatures..$^{40}$ The carrier concentration $n_{T S S}$, can be obtained using following relationship between sheet conductance and $\mu$, as, $G_{T S S}=G(\omega \rightarrow 0)=n_{T S S} . e . \mu$, yielding a value of $\sim 6 \times 10^{12} \mathrm{~cm}^{-2}$ at $4 \mathrm{~K}$. This is consistent with the surface state carrier concentration measured by Brahlek et al. ${ }^{23}$; they obtained the surface carrier density to be $\sim 5 \times 10^{12} \mathrm{~cm}^{-2}$ from Shubnikov-de Haas $(\mathrm{SdH})$ oscillations, suggesting the TSS origin of the charge carriers in these measurements. Assuming a linear dispersion of the topological surface state, we estimated the position of the Fermi level, given by $E_{F}=$ $\hbar v_{F} \sqrt{4 \pi n_{T S S}}$ (where $v_{F}$ is the Fermi velocity) to be $\sim(0.38 \pm 0.17) \mathrm{eV}$ above the Dirac point. This value is consistent with the ARPES reports ${ }^{6,15,16}$, further suggesting that the sheet carrier density obtained from the THz measurements is in fact representative of the surface electron density at $4 \mathrm{~K}$. The THz optical carrier density $\left(n_{2 \mathrm{D}}\right)$ rises with temperature (Figure $5 b$ ), indicating an increasing contribution from the bulk carriers to the measured conduction clearly observed above $50 \mathrm{~K}$. This is in good agreement with bulk carrier densities of $\sim 5 \times 10^{14} \mathrm{~cm}^{-2}$ measured by He et al. ${ }^{27}$ The carrier density obtained from Hall measurements show a predominantly bulk/ impurity response, possibly due to surface doping from the device processing involved, ${ }^{37}$ as discussed below. The measured THz sheet carrier density of TSS $n_{T S S}=k_{F, T S S}{ }^{2} /(4 \pi)$, yield a Fermi wave vector $k_{F, T S S}$ of $0.09 \AA^{-1}$ at $4 \mathrm{~K}$ and $0.11 \AA^{-1}$ at $15 \mathrm{~K}$, consistent with the ARPES values associated with topological surface bands on $\mathrm{Bi}_{2} \mathrm{Se}_{3}$ films. ${ }^{6,15,16}$ This further supports the TSS origin of the carrier densities and mobilities. It should be noted that our THz measurement stands out as a technique to probe both the surface and bulk states, though it is more surface-sensitive at higher frequencies due to a reduced skin depth. A comparison of the Hall mobilities obtained from the samples processed using the chemical etch solution with the $\mathrm{Ar}^{+}$ion milling is also shown in the Figure 5a. The mobility worsens in the $\mathrm{Bi}_{2} \mathrm{Se}_{3}$ samples, which were processed using the $\mathrm{Ar}^{+}$ion milling compared to samples which were processed using the wet chemical etch method. This could be attributed to the increase in the density of defect states in $\mathrm{Bi}_{2} \mathrm{Se}_{3}$ after being exposed to the ion milling process, due to increased disorder in the system. ${ }^{45}$

We note that the values of the carrier density obtained from the transport measurements at temperature below $50 \mathrm{~K}$ are higher compared to the values obtained from the $\mathrm{THz}$ measurements as shown in Figure 5b. Moreover, the transport mobility values obtained from the Hall measurements are significantly lower at temperatures $<50 \mathrm{~K}$, compared to the $\mathrm{THz}$ measurements. The carrier densities obtained from samples processed with $\mathrm{Ar}^{+}$ion milling were higher than the chemically etched samples. This suggests that device processing effects the mobility of topological insulators while increasing the overall doping of the TI film. This can be understood as follows. The device processing into Hall bars involves use of various chemical (such as MF 319 developer [tetramethylammonium hydroxide]) and DI water. This could modify the surface of the TI with the possible reaction to occur at the surface

$$
\mathrm{Bi}_{2} \mathrm{Se}_{3}+6 \mathrm{H}_{2} \mathrm{O} \rightarrow 3 \mathrm{H}_{2} \mathrm{Se} \uparrow+2 \mathrm{Bi}(\mathrm{OH})_{3}
$$

The $\mathrm{H}_{2} \mathrm{Se}$ gas escapes, leaving Se vacancies ${ }^{41}$ and bismuth hydroxide behind ${ }^{42}$, which cause $n$-doping of the $\mathrm{Bi}_{2} \mathrm{Se}_{3}$ film. Even though this does not directly affect the TSS, the increased doping makes it more challenging to distinguish the surface response from the bulk. ${ }^{43}$

This potentially highlights the well-known issue associated with trying to distinguish TSS contributions from a noisy bulk background through transport measurements, especially for ultra-thin films ${ }^{29}$. The low frequency extrapolation of the $\mathrm{THz}$ conductance spectra should approach the d.c. conductance values obtained from standard transport measurements. However, it should be noted that standard transport measurements rely heavily on device geometry and processing, making it difficult to obtain a TSS response from the pristine surface of a TI. While we took all precautions to minimize any direct surface contact, the DI water involved in the device processing could react with the $\mathrm{Bi}_{2} \mathrm{Se}_{3}$ to form hydrogen selenide and bismuth hydroxide resulting in an overall $n$-type behavior of the film. ${ }^{41}$ Even though this does not directly affect the TSS, the increased doping might make it more challenging to distinguish surface from bulk response. The THz conductance measurements, on the other hand, present an alternative way of obtaining the quasi-d.c. conductance behavior in TIs.

Although the transport measurements reveal useful parameters such as $\alpha \approx 0.5$ and $L_{\varphi} \approx 380 \mathrm{~nm}$, consistent with previous studies on $\mathrm{Bi}_{2} \mathrm{Se}_{3}{ }^{44}$ and other TIs ${ }^{35}$, the magneto-transport response could be due to coupling of the top and bottom TSS through the bulk or could be due to a significant response from the top TSS. In both cases, however, $\alpha=0.5$, is consistent with the presence of topological surface state(s), though we can't exclude the possibility of bulk (or quantum well state) interference in the transport measurements. As the Hall transport mobility in our case is low, we estimate the magnetic field would have to be raised to $\approx 18 \mathrm{~T}$ to observe the first $\mathrm{SdH}$ oscillation (from the condition $\mu B>1$ for closed orbits, Hall transport mobility is $552 \mathrm{~cm}^{2} / \mathrm{V} \cdot \mathrm{s}$ ), which could provide further information about the surface transport. The THz 
Return to the Manage Active Submissions page at http://spie.org/submissions/tasks.aspx and approve or disapprove this submission. Your manuscript will not be published without this approval. Please contact author_help@spie.org with any questions or concerns.

measurements on the other hand, clearly shows a TSS response at low temperature and a gradual transition to the bulk and QWS at temperatures $>30 \mathrm{~K}$, demonstrating the surface sensitive advantage provided by the $\mathrm{THz}$ measurement over the transport measurement.

\section{CONCLUSION}

In conclusion, we used THz spectroscopy and magneto-transport to provide evidence for the existence of a TSS at low temperature in a 23-QL-thick $\mathrm{Bi}_{2} \mathrm{Se}_{3}$ film. The temperature dependence of the $\mathrm{THz}$ mobility and scattering lifetime demonstrates a metallic behavior consistent with the presence of a metallic TSS below $30 \mathrm{~K}$, reaching a mobility value of $\sim 2100 \mathrm{~cm}^{2} / \mathrm{V}$.s and $0.18 \mathrm{ps}$ respectively, at $4 \mathrm{~K}$. The mobility trend further elucidated the bulk-to-surface relaxation mechanism which suppresses the increase of the surface scattering rate above $50 \mathrm{~K}$. In addition, we compared the Hall mobility and carrier density in $\mathrm{Bi}_{2} \mathrm{Se}_{3}$ samples processed using $\mathrm{Ar}^{+}$ion milling and wet chemical etching. We observed an overall reduction in the Hall mobility of samples processed with $\mathrm{Ar}^{+}$ion milling compared to chemically etched samples. This was attributed to an increase in the density of defect states, due to introduction of disorder from the $\mathrm{Ar}^{+}$ion milling. We further show that magneto-transport reveals WAL at low temperatures giving $\alpha \approx 0.5$ which indicates coupling of the top and bottom TSS. The capability of THz spectroscopy to discriminate surface state from bulk behavior demonstrates the strength of the technique in contrast to standard Hall measurements. The Fermi level is estimated to be around $0.38 \mathrm{eV}$ above the Dirac point with the Fermi wave vector $\sim 0.1 \AA^{-1}$, consistent with ARPES measurements on $\mathrm{Bi}_{2} \mathrm{Se}_{3}$. The direct coupling between the THz optical radiation and the TSS opens up the possibility of developing novel TI devices such as room temperature photodetectors and $\mathrm{THz}$ modulators operational in the $\mathrm{THz}$ gap. Our work presents a non-destructive method to explore their properties at $\mathrm{THz}$ frequencies in a contact-free manner.

\section{ACKNOWLEDGMENTS}

The authors of this manuscript acknowledge financial support from the Engineering and Physical Sciences Research Council (EPSRC), grant numbers KLZA/145 - High precision terahertz spectroscopy and microscopy (HyperTerahertz) and the Cambridge Commonwealth Trust.

\section{REFERENCES}

[1] Hasan, M. Z.; Kane, C. L. Colloquium: Topological insulators. Rev. Mod. Phys. 2011, 82, 3045-3067.

[2] Hasan, M. Z.; Moore, J. E. Three-Dimensional Topological Insulators. Annu. Rev. Condens. Matter Phys. 2011, 2, 55-78.

[3] Qi, X. L.; Zhang S. C. Topological insulators and superconductors. Rev. Mod. Phys. 2011, 83, 1057.

[4] Fu. L,; Kane, C. L; Mele, E. J. Topological Insulators in Three Dimensions. Phys. Rev. Lett. 2007, 98, 106803.

[5] Moore, J. E.; Balents. L. Topological invariants of time-reversal-invariant band structures. Phys. Rev. B. 2007, 75, 121306.

[6] Xia, Y.; Qian, D.; Hsieh, D.; Wray, L.; Pal, A.; Lin, K.; Bansil, A.; Grauer, A.; Cava, R. J.; Hasan, M. Z. Observation of a large-gap topological-insulator class with a single Dirac cone on the surface. Nat. Phys. 2009, 5, 398-402.

[7] Zhang, H.; Liu, C. X.; Qi, X.-L.; Dai, X.; Fang, Z.; Zhang, S. C. Topological Insulators in $\mathrm{Bi}_{2} \mathrm{Se}_{3}, \mathrm{Bi}_{2} \mathrm{Te}_{3}$ and $\mathrm{Sb}_{2} \mathrm{Te}_{3}$ with a Single Dirac Cone on the Surface. Nat. Phys. 2009, 5, 438-442.

[8] Hsieh, D.; Xia, Y.; Qian, D.; Wray, L.; Meier, F.; Dil, J.H.; Osterwalder, J.; Patthey, L.; Fedorov, A.V.; Lin, H.; Bansil, A.; Grauer, D.; Hor, Y. S; Cava, R. J.; Hasan, M. Z. Observation of Time-Reversal-Protected SingleDirac-Cone Topological-Insulator States in $\mathrm{Bi}_{2} \mathrm{Se}_{3}$ and $\mathrm{Sb}_{2} \mathrm{Te}_{3}$. Phys. Rev. Lett. 2009, 103, 146401.

[9] Chen, Y. L.; Analytis, J. G.; Chu, J. H.; Liu, Z. K.; Mo, S. K.; Qi, X. L.; Zhang, H. J.; Lu, D. H.; Dai, X.; Fang, Z.; Zhang, S. C.; Fisher, I. R.; Hussain, Z.; Shen, Z. X. Experimental Realization of a Three-Dimensional Topological Insulator, $\mathrm{Bi}_{2} \mathrm{Te}_{3}$. Science 2009, 325, 178-181.

[10] Kane, C. L.; Mele, E. J. Z 2 Topological Order and the Quantum Spin Hall Effect. Phys. Rev. Lett. 2005, 95, 146802. 
Return to the Manage Active Submissions page at http://spie.org/submissions/tasks.aspx and approve or disapprove this submission. Your manuscript will not be published without this approval. Please contact author_help@spie.org with any questions or concerns.

[11] Liu, C. X.; Qi, X. L.; Zhang, H. J.; Dai, X.; Fang, Z.; Zhang, S. C. Model Hamiltonian for topological insulators. Phys. Rev. B. 2010, 82, 045122.

[12] Fu, L.; Kane, C. L. Superconducting Proximity Effect and Majorana Fermions at the Surface of a Topological Insulator. Phys. Rev. Lett. 2008, 100, 096407.

[13] Hsieh, D.; Qian, D.; Wray, L.; Xia, Y.; Hor, Y. S.; Cava, R. J.; Hasan, M. Z. A topological Dirac insulator in a quantum spin Hall phase. Nature 2008, 452, 970-974.

[14] Brahlek, M.; Kim, Y. S.; Bansal, N.; Edrey, E.; Oh, S. Surface versus bulk state in topological insulator $\mathrm{Bi}_{2} \mathrm{Se}_{3}$ under environmental disorder. Appl. Phys. Lett. 2011, 99, 012109.

[15] Bianchi, M.; Guan, D.; Bao, S.; Mi, J.; Iversen, B.; King, P.; Hofmann, P. Coexistence of the topological state and a two-dimensional electron gas on the surface of $\mathrm{Bi}_{2} \mathrm{Se}_{3}$. Nat. Commun. 2010, 1, 128.

[16] King, P. D. C.; Hatch, R. C.; Bianchi, M.; Ovsyannikov, R.; Lupulescu, C.; Landolt, G.; Slomski, B.; Dil, J. H.; Guan, D.; Mi, J. L.; Rienks, E. D. L.; Fink, J.; Lindblad, A.; Svensson, S.; Bao, S.; Balakrishnan, G.; Iversen, B. B.; Osterwalder, J.; Eberhardt, W.; Baumberger, F.; Hofmann, P. Large Tunable Rashba Spin Splitting of a TwoDimensional Electron Gas in $\mathrm{Bi}_{2} \mathrm{Se}_{3}$. Phys. Rev. Lett. 2011, 107, 096802.

[17] Valdés Aguilar, R.; Qi, J.; Brahlek, M.; Bansal, N.; Azad, A.; Bowlan, J.; Oh, S.; Taylor, A. J.; Prasankumar, R. P.; Yarotski, D. A. Time-Resolved Terahertz Dynamics in Thin Films of the Topological Insulator $\mathrm{Bi}_{2} \mathrm{Se}_{3} . A p p l$. Phys. Lett. 2015, 106, 011901.

[18] Tang, C. S.; Xia, B.; Zou, X.; Chen, S.; Ou, H. W.; Wang, L.; Rusydi, A.; Zhu, J. X.; Chia, E. E. M. Terahertz Conductivity of Topological Surface States in $\mathrm{Bi}_{1.5} \mathrm{Sb}_{0.5} \mathrm{Te}_{1.8} \mathrm{Se}_{1.2 .}$. Sci. Rep. 2013, 3, 3513.

[19] Yashina, L.; Sánchez-Barriga, J.; Scholz, M. R.; Volykhov, A. A.; Sirotina, A. P.; Neudachina, V. S.; Tamm, M. E.; Varykhalov, A.; Marchenko, D.; Springholz, G.; Bauer, G.; Knop-Gericke, A.; Rader. O. Negligible Surface Reactivity of Topological Insulators $\mathrm{Bi}_{2} \mathrm{Se}_{3}$ and $\mathrm{Bi}_{2} \mathrm{Te}_{3}$ Towards Oxygen and Water. ACS Nano 2013, 7, 5181.

[20] Qi, J.; Chen, X.; Yu, W.; Cadden-Zimansky, P.; Smirnov, D.; Tolk, N. H.; Miotkowski, I.; Cao, H.; Chen, Y. P.; Wu, Y.; Qiao, S.; Jiang, Z. Ultrafast Carrier and Phonon Dynamics in $\mathrm{Bi}_{2} \mathrm{Se}_{3}$ Crystals. Appl. Phys. Lett. 2010, 97, 182102.

[21] Nuss, M. C.; Orenstein, J.; Millimeter and Submillimeter Wave Spectroscopy of Solids. Springer-Verlag Berlin Heidelberg, 1998; pp 7-50.

[22] Analytis, J. G.; Chu, J. H.; Chen, Y.; Corredor, F.; McDonald, R. D.; Shen, Z. X.; Fisher, I. R. Bulk Fermi Surface Coexistence with Dirac Surface State in $\mathrm{Bi}_{2} \mathrm{Se}_{3}$ : A Comparison of Photoemission and Shubnikov-de Haas Measurements. Phys. Rev. B. 2010, 81, 205407.

[23] Brahlek, M.; Koirala, N.; Salehi, M.; Bansal, N.; Oh, S. Emergence of Decoupled Surface Transport Channels in Bulk Insulating $\mathrm{Bi}_{2} \mathrm{Se}_{3}$ Thin Films. Phys. Rev. Lett. 2014, 113, 026801.

[24] Wray, L. A.; Xu, S.; Xia, Y.; Hor, Y. S.; Qian, D.; Fedorov, A. V; Lin, H.; Bansil, A.; Cava, R. J.; Hasan, M. Z. Observation of Topological Order in a Superconducting Doped Topological Insulator. Nat. Phys. 2010, 6, 855859.

[25] Yu, P. Y.; Cardona, M. Fundamentals of Semiconductors: Physics and Materials Properties, 3rd ed.; SpringerVerlag: Berlin Heidelberg, 2010; pp 220-223.

[26] Park, B. C.; Kim, T. H.; Sim, K. I.; Kang, B.; Kim, J. W.; Cho, B.; Jeong, K. H.; Cho, M. H.; Kim, J. H. Terahertz Single Conductance Quantum and Topological Phase Transitions in Topological Insulator $\mathrm{Bi}_{2} \mathrm{Se}_{3}$ Ultrathin Films. Nat. Commun. 2015, 6, 6552.

[27] He, L.; Xiu, F.; Yu, X.; Teague, M.; Jiang, W.; Fan, Y.; Kou, X.; Lang, M.; Wang, Y.; Huang, G.; Nai-Chang, Y.; Wang, K. L. Surface-Dominated Conduction in a 6-nm thick $\mathrm{Bi}_{2} \mathrm{Se}_{3}$ Thin Film. Nano Lett. 2012, 12, 14861490.

[28] Kong, D.; Chen, Y.; Cha, J.; Zhang, Q.; Analytis, J.; Lai, K.; Liu, Z.; Hong, S.; Koski, K.; Mo, S. et al. Ambipolar Field Effect in the Ternary Topological Insulator $\left(\mathrm{Bi}_{\mathrm{x}} \mathrm{Sb}_{1-\mathrm{x}}\right)_{2} \mathrm{Te}_{3} \mathrm{By}$ Composition Tuning. Nature Nanotechnology 2011, 6, 705-709.

[29] Bansal, N.; Kim, Y. S.; Brahlek, M.; Edrey, E.; Oh, S. Thickness-Independent Transport Channels in Topological Insulator $\mathrm{Bi}_{2} \mathrm{Se}_{3}$ Thin Films. Phys. Rev. Lett. 2012, 109, 116804.

[30] Chen, J.; He, X. Y.; Wu, K. H.; Ji, Z. Q.; Lu, L.; Shi, J. R.; Smet, J. H.; Li, Y. Q. Tunable Surface Conductivity in $\mathrm{Bi}_{2} \mathrm{Se}_{3}$ Revealed in Diffusive Electron Transport. Phys. Rev. B. 2011, 83, 241304.

[31] Hikami, S.; Larkin, A. I.; Nagaoka, Y. Spin-Orbit Interaction and Magnetoresistance in the Two-Dimensional Random System. Prog. Theor. Phys. 1980, 63, 707-710.

[32] Steinberg, H.; Lalo, J.; Fatemi, V.; Moodera, J. S.; Herrero, P. J. Electrically Tunable Surface-to-Bulk Coherent Coupling in Topological Insulator Thin Films. Phys. Rev. Lett. 2011, 84, 233101. 
Return to the Manage Active Submissions page at http://spie.org/submissions/tasks.aspx and approve or disapprove this submission. Your manuscript will not be published without this approval. Please contact author_help@spie.org with any questions or concerns.

[33] Veldhorst, M.; Snelder, M.; Hoek, M.; Molenaar, C. G.; Leusink, D. P.; Golubov, A. A.; Hilgenkamp, H.; Brinkman, A. Magnetotransport and Induced Superconductivity in Bi Based Three-Dimensional Topological Insulators. Phys. Status Solidi - Rapid Res. Lett. 2013, 7, 26-38.

[34] He, H. T.; Wang, G.; Zhang, T.; Sou, I. K.; Wong, G. K. L.; Wang, J. N.; Lu, H. Z.; Shen, S. Q.; Zhang, F. C. Impurity Effect on Weak Antilocalization in the Topological Insulator $\mathrm{Bi}_{2} \mathrm{Te}_{3}$. Phys. Rev. Lett. 2011, 106, 166805.

[35] Nguyen, T.; Backes, D.; Singh, A.; Mansell, R.; Barnes, C.; Ritchie, D.; Mussler, G.; Lanius, M.; Grützmacher, D.; Narayan, V. Topological States and Phase Transitions in $\mathrm{Sb}_{2} \mathrm{Te}_{3}-\mathrm{GeTe}$ Multilayers. Sci. Rep. 2016, 27716.

[36] Sobota, J. A.; Yang, S.; Analytis, J. G.; Chen, Y. L.; Fisher, I. R.; Kirchmann, P. S.; Shen, Z. X. Ultrafast Optical Excitation of a Persistent Surface-State Population in the Topological Insulator $\mathrm{Bi}_{2} \mathrm{Se}_{3}$. Phys. Rev. Lett. 2012, 108, 117403.

[37] Hajlaoui, M.; Papalazarou, E.; Mauchain, J.; Lantz, G.; Moisan, N.; Boschetto, D.; Jiang, Z.; Miotkowski, I.; Chen, Y. P.; Taleb-Ibrahimi, A; Perfetti. L.; Marsi. M. Ultrafast Surface Carrier Dynamics in the Topological Insulator $\mathrm{Bi}_{2} \mathrm{Te}_{3}$. Nano Lett. 2012, 12, 3532-3536.

[38] Wang, Y. H.; Hsieh, D.; Sie, E. J.; Steinberg, H.; Gardner, D. R.; Lee, Y. S.; Jarillo-Herrero, P.; Gedik, N. Measurement of Intrinsic Dirac Fermion Cooling on the Surface of the Topological Insulator $\mathrm{Bi}_{2} \mathrm{Se}_{3}$ Using Time-Resolved and Angle-Resolved Photoemission Spectroscopy. Phys. Rev. Lett. 2012, 109, 127401.

[39] Sim, S.; Brahlek, M.; Koirala, N.; Cha, S.; Oh, S.; Choi, H. Ultrafast Terahertz Dynamics of Hot Dirac-Electron Surface Scattering in the Topological Insulator $\mathrm{Bi}_{2} \mathrm{Se}_{3}$. Phys. Rev. B. 2014, 89, 165137.

[40] Butch, N. P.; Kirshenbaum, K.; Syers, P.; Sushkov, A. B.; Jenkins, G. S.; Drew, H. D.; Paglione, J. Strong Surface Scattering in Ultrahigh-Mobility $\mathrm{Bi}_{2} \mathrm{Se}_{3}$ Topological Insulator Crystals. Phys. Rev. B. 2010, 81, 241301.

[41] Benia, H. M.; Lin, C.; Kern, K.; Ast, C. R. Reactive Chemical Doping of the $\mathrm{Bi}_{2} \mathrm{Se}_{3}$ Topological Insulator. Phys. Rev. Lett. 2011, 107, 177602.

[42] Hor, Y.; Richardella, A.; Roushan, P.; Xia, Y.; Checkelsky, J.; Yazdani, A.; Hasan, M.; Ong, N.; Cava, R. pType $\mathrm{Bi}_{2} \mathrm{Se}_{3}$ for Topological Insulator and Low-Temperature Thermoelectric Applications. Physical Review B, 2009, 79.

[43] Kamboj, V.; Singh, A.; Ferrus, T.; Beere, H.; Duffy, L.; Hesjedal, T.; Barnes, C.; Ritchie, D. Probing the Topological Surface State in $\mathrm{Bi}_{2} \mathrm{Se}_{3}$ Thin Films Using Temperature-Dependent Terahertz Spectroscopy. ACS Photonics 2017, 4, 2711-2718.

[44] Chen, J.; Qin, H. J.; Yang, F.; Liu, J.; Guan, T.; Qu, F. M.; Zhang, G. H.; Shi, J. R.; Xie, X. C.; Yang, C. L.; Wu, K. H.; Li, Y. Q.; Lu. L. Gate-Voltage Control of Chemical Potential and Weak Antilocalization in $\mathrm{Bi}_{2} \mathrm{Se}_{3}$. Phys. Rev. Lett. 2010, 105, 176602.

[45] Banerjee, K.; Son, J.; Deorani, P.; Ren, P.; Wang, L.; Yang, H. Defect-Induced Negative Magnetoresistance and Surface State Robustness in the Topological Insulator BiSbTeSe 2. Physical Review B, 2014, 90, 235427.

[46] Kamboj, V.; Singh, A.; Ferrus, T.; Beere, H.; Duffy, L.; Hesjedal, T.; Barnes, C.; Ritchie, D. Temperature evolution of topological surface states in $\mathrm{Bi}_{2} \mathrm{Se}_{3}$ thin films studied using terahertz spectroscopy. Proc. of SPIE 2017, 10103, 101030D-1. 